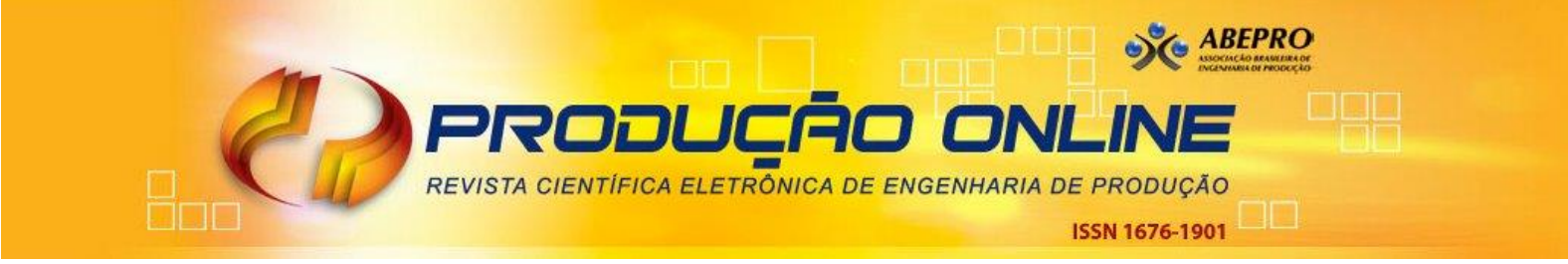

\title{
ECONOMIA CIRCULAR: O CASO DOS RESÍDUOS DA CONSTRUÇÃO CIVIL CARIRIENSE
}

\section{CIRCULAR ECONOMY: THE CASE OF WASTE FROM CIVIL CONSTRUCTION CARIRIENSE}

\author{
Josivan Leite Alves *E-mail: josivanleite3@gmail.com \\ Igor Bernardino Borges * E-mail: igor.bb7@gmail.com \\ Mayara Alves Ramos * E-mail: mayramosadm@gmail.com \\ Jeniffer de Nadae *E-mail: jeniffer.nadae@ufca.edu.br \\ Rebeca da Rocha Grangeiro * E-mail: rebeca.grangeiro@ufca.edu.br \\ *Universidade Federal do Cariri (UFCA), Juazeiro do Norte-CE
}

Resumo: A aplicação da economia circular no setor construtivo propõe uma configuração produtiva que pode diminuir o potencial degradador de atividades desenvolvidas nesta área. Os conceitos de Economia Circular (EC) e de Sustentabilidade buscam promover a inter-relação entre o sistema econômico e o meio ambiente, ao se preocuparem com o destino dos resíduos sólidos. Neste sentido, esta pesquisa tem por objetivo analisar os princípios da economia circular no setor da construção civil do cariri cearense. Para tanto, foram realizados dois estudos de caso, sendo um deles em uma fabricante e distribuidora de gesso acartonado e o outro em uma fabricante e instaladora de esquadrias de alumínio. As empresas revelaram elevado grau de preocupação com o meio ambiente e, a partir das informações coletadas, foi possível desenvolver um fluxograma da cadeia produtiva destas. Por fim, observou-se que a economia circular estabelece um equilíbrio entre a dinâmica da lucratividade e o meio ambiente ao reduzir os desperdícios, e percebeu-se que, para implementar o conceito, é necessário que haja interesse tanto dos empresários quanto dos consumidores. Espera-se que este estudo contribua para ampliar a compreensão do conceito de EC e de sua importância para o setor da construção civil, minimizando, assim, a quantidade de resíduos descartados no meio ambiente.

Palavras-chave: Economia Circular. Sustentabilidade. Demolição. Resíduos. Construção Civil.

Abstract: The application of the Circular Economy (CE) in the construction sector proposes a productive configuration that can diminish the degrading potential of activities developed in this area. The concepts of the Circular Economy and Sustainability seeks to promote the interrelationship between the economic system and the environment, as it is concerned with the destination of Solid Waste. In this sense, the purpose of this research is to analyze the principles of the Circular Economy in the Cariri cearense civil construction sector. For that, two case studies were carried out, one in a Manufacturer and distributor of gypsum plasterboard and the other is a manufacturer and installer of aluminum frames. The companies showed a high degree of concern with the environment and from the information collected it was possible to develop a flowchart of the production chain by each company. Finally, it was observed that the CE establishes a balance between the dynamics of profitability and the environment by reducing the waste and realized that, implement the concept, it is necessary that the interest of both entrepreneurs and consumers. It is hoped that this study will contribute to an understanding of the concept of CE and its importance for the construction industry, minimizing, like this, the amount of waste in the environment.

Keywords: Circular Economy. Sustainability. Demolition. Waste. Civil Construction. 


\section{INTRODUÇÃO}

O modelo econômico de produção atual gira em torno da cultura do desperdício, na qual os materiais que entram na cadeia produtiva são descartados sem possibilidade de retorno, conhecido por modelo de Economia Linear (LI). Ademais, conciliar uma construção lucrativa e que promova poucos danos à natureza tem se tornado uma das maiores dificuldades no campo da engenharia civil, pois a maioria dos resíduos gerados por esta são de difícil reaproveitamento ou possuem custo elevado para reciclagem ou reutilização.

Mesmo sabendo que os recursos naturais do planeta são finitos, a Economia Linear se baseia na priorização dos aspectos econômicos, desconsiderando as orientações ecológicas e sociais, elevando os índices de poluição (SAUVÉ; BERNARD; SLOAN, 2016).

As iniciativas que garantam um desenvolvimento sustentável na construção civil fazem-se necessárias pois, de acordo com dados do IBGE (2017), o custo nacional da construção, por metro quadrado, passou de $R \$ 1.064,76$ para $R \$$ 1.066,68 em novembro de 2017, sendo $R \$ 544,97$ relativos aos materiais e $R \$$ 521,71 à mão de obra. Com isso, percebe-se que os materiais de construção elevam consideravelmente o custo final da obra, já que fazem parte de cerca de $51,09 \%$ do custo total. Além disso, há uma elevada produção de resíduos sólidos, que configurou uma coleta de 45,1 milhões de toneladas de Resíduos de Construção e Demolição (RCD) em 2016 (ABRELP, 2016). Tal fato gera a proporcionalidade de que uma tonelada de lixo urbano produzido equivale a duas toneladas de entulhos da construção civil (NETO, 2005).

Diante disso, os fundamentos da Economia Circular (EC) estão atraindo a atenção do mundo, trazendo novas perspectivas no que diz respeito à produção tradicional. A EC considera os padrões do modelo produtivo linear como arcaicos e ineficientes, além do fato de que ela respeita os quesitos de sustentabilidade, dando ênfase mais à efetividade do que à eficiência (NOYA et al., 2017; VELTE; STEINHILPER, 2016).

Assim, a EC implementa uma nova configuração e ciclo de vida dos materiais dentro da cadeia produtiva, atendendo a aspectos sociais, econômicos e ambientais. A EC propõe uma nova perspectiva de gerir os resíduos em que estes são projetados e otimizados para retornar à cadeia de produção, opondo-se ao conceito Revista Produção Online. Florianópolis, SC, v. 20, n. 2, p. 449-471, 2020 
de eliminação e até mesmo reciclagem que incorporam grandes quantidades de energia e mão de obra (ELLEN MACARTHUR FOUNDATION, 2013).

De acordo com Araújo e Queiroz (2017), a EC pode ser considerada um ciclo de desenvolvimento contínuo que preserva e aprimora o capital natural, otimizando a produção de recursos, minimizando riscos sistêmicos, administrando estoques finitos e fluxos renováveis, oferecendo diversos mecanismos de criação de valor dissociados do consumo de recursos finitos. O modelo proposto pela EC aproxima um novo paradigma econômico e implica em uma nova forma de fazer produtos desde o seu desenvolvimento, permitindo construir negócios que atendam ao crescimento econômico e considerem: a sustentabilidade ambiental, a diminuição dos riscos inerentes à volatilidade, às incertezas dos preços das matérias-primas e dos recursos energéticos (LETT, 2014), gerando benefícios ambientais, sociais e econômicos.

Nesse sentido, as características regenerativas da EC possibilitam que a entrada de recursos e o desperdício sejam minimizados pela desaceleração, fechamento e estreitamento de loops de material e energia (GEISSDOERFER et al., 2017; ELLEN MACARTHUR FOUNDATION, 2013). Por meio de um design de produto duradouro, manutenção, reparo, reutilização, remanufatura, remodelação e reciclagem é possível que haja uma redução na dissipação de energia do sistema, tornando-o mais eficiente. (GEISSDOERFER et al., 2017). Vale ressaltar que segundo Hilsdorf et al. (2019) a indústria da remanufatura engloba os aspectos desde econômicos, a ambientais e abrange diversos setores das indústria, porém há ausência de regras especificas.

Assim sendo, o presente trabalho tem por finalidade analisar os princípios da EC no setor da construção civil do Cariri cearense, por meio de dois estudos de caso, sendo um em uma fabricante e distribuidora de gesso acartonado e o outro em uma fabricante e instaladora de esquadrias de alumínio.

Acrescenta-se que a construção civil consome $50 \%$ da produção de aço no mundo e 3 bilhões de toneladas de matéria-prima são utilizadas por ano na fabricação de materiais de construção, fazendo com que o setor seja o maior consumidor dos recursos naturais (WORLD ECONOMIC FORUM, 2016). Sendo que tal consumo deve ser realizado sem o comprometimento das interdependências entre seres vivos e meio ambiente, cabendo as empresas promover atitudes que Revista Produção Online. Florianópolis, SC, v. 20, n. 2, p. 449-471, 2020 
valorizem o meio ambiente (FLORIM; QUELHAS, 2005). Deste modo, são necessárias melhorias na administração do uso de concreto, alvenaria (tijolos e blocos de concreto), argamassa e resíduos de cerâmica, pois, são estes que compõem $90 \%$ dos resíduos de construção e demolição na China e têm elevado potencial de reciclagem (DUANE LI, 2016).

Este estudo encontra-se dividido em 6 seções: a introdução (1) aborda a apresentação ao tema. O referencial teórico (2) atenta sobre os conceitos principais acerca da economia circular na construção civil e quais são seus principais aspectos em pesquisas, referenciados por autores da área. Em seguida, a metodologia (3) relata os parâmetros utilizados para que o estudo fosse realizado, suas principais métricas e critérios, aliando coleta de dados de arquivos, entrevistas, questionários e observação in loco. Já os resultados e as discussões (4) retratam quais as principais considerações das organizações pesquisadas sobre a utilização do conceito de EC, observando principalmente, por parte dos entrevistados, a preocupação destes com o impacto gerado ao meio ambiente pela sua atividade produtiva, além de se investigar se os entrevistados demonstram conhecimento sobre do tema e o utilizam com propriedade. Na conclusão (5), as principais considerações e limitações do estudo são relatadas, como forma de explanação dos resultados obtidos e se estes respondem ao objeto de estudo.

\section{ECONOMIA CIRCULAR E A CONSTRUÇÃO CIVIL}

A Economia Circular (EC) funciona de forma efetiva em qualquer escala, caracterizando-se como restaurativa e regenerativa, mantendo seus produtos em elevado patamar de uso e valor, promovendo uma continuidade em sua configuração e fazendo a manutenção do desenvolvimento sustentável que garante a preservação dos recursos naturais (ELLEN MACARTHUR FOUNDATION, 2015). Noya et al. (2017) asseguram que a EC se define pelo baixo consumo de materiais e recursos no regime de produção, permitindo máximo aproveitamento desses recursos com foco em uma eficiência global.

A sustentabilidade requer significativas mudanças nos modos de produção, consumo e comportamento atuais da sociedade. Dessa forma, é preciso que sejam desenvolvidos mecanismos por meio dos quais conceitos possam ser transformados

Revista Produção Online. Florianópolis, SC, v. 20, n. 2, p. 449-471, 2020 
em ação, para que haja a mudança de uma economia ineficiente em consumo de recursos para uma onde o consumo de matérias-primas seja racionalizado, permitindo a transformação de uma EL para uma EC. A Figura 1 exemplifica ambos os modelos de produção.

Figura 1 - Contrastando os conceitos de Economia Linear e Economia Circular

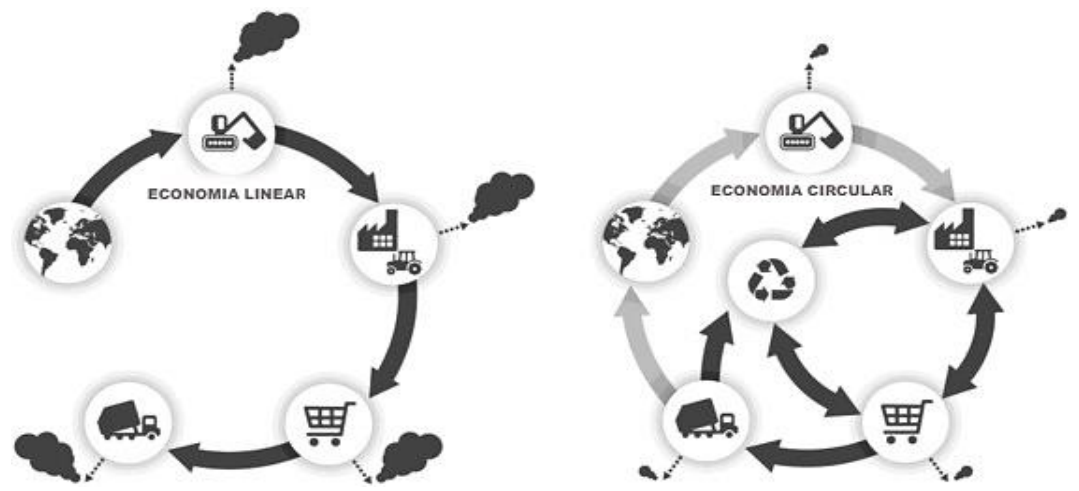

Fonte: Adaptado de Sauvé, Bernard e Sloan (2016, p. 5).

Uma EL é definida como a conversão de recursos naturais em resíduos por meio da produção (MURRAY, SKENE; HAYNES, 2015). A produção de resíduos ocasiona a deterioração da natureza através da remoção do capital natural do meio ambiente e da redução do valor do capital natural, ocasionada pela poluição gerada pelo descarte de resíduos.

A dinâmica da EL coincide com o "sistema linear" de Stahel (1982). Nesse sistema, os bens produzidos obedecem à lógica produção-consumo-descarte, o que proporciona "deterioração ambiental embutida em ambas as extremidades" (STAHEL, 1982, p. 73).

O setor da construção civil corresponde a um dos maiores geradores de resíduos sólidos no mundo. Então, faz-se necessária, uma evolução no que diz respeito aos processos, componentes e sistemas para evitar desperdícios e elevar a eficiência (ARUP, 2016).

De acordo com Ellen MacArthur Foundation (2017), os princípios da EC, tal qual aplicados no setor de edifícios e construção, incorporam todos os aspectos da criação de um edifício. Arup (2016) afirma que eles incluem construção modular, pré-fabricada e fora do local, projeto de reforma, reutilização e reciclagem de materiais e concepção de resíduos. Assim sendo, uma construção bem-sucedida em termos de sustentabilidade, atendendo aos conceitos estabelecidos pela EC, está 
estritamente relacionada com a qualidade da definição do projeto na fase de planejamento (SANCHEZ; HAAS, 2018).

$A$ transição da EL para EC na indústria da construção civil é fundamental, já que este é o setor que mais degrada o meio ambiente (POMPONI; MONCASTER, 2017). Os edifícios são compostos de produtos manufaturados e que geralmente são resultados de um único e bem definido projeto, tornando-o complexo, onde cada material interage no espaço e no tempo e tem seu próprio ciclo de vida (POMPONI; MONCASTER, 2017). O desperdício gerado pela Economia Linear nos ambientes construtivos representa perdas significativas de valiosos minerais, metais e materiais orgânicos que poderiam ser remanufaturados se os fundamentos da $E C$ fossem bem aplicados (WORLD ECONOMIC FORUM, 2016).

As obras são definidas principalmente em três atividades: construção, renovação e demolição (COCHRAN; TOWNSEND, 2010). Os Resíduos de Construção e Demolição (RCD) são os desperdícios de materiais gerados durante o ciclo de construção de uma edificação, conforme Figura 2 (ESA; HALOG; RIGAMONTI, 2016).

Figura 2 - Fluxo de materiais gerados durante o ciclo de vida de diferentes construções civis

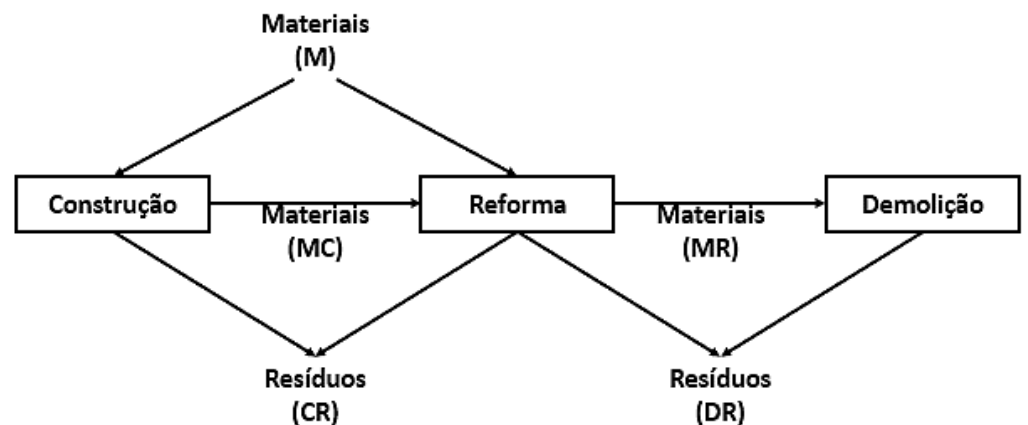

Fonte: Adaptado de Cochran; Townsend (2010, p. 2248).

Os RCD apresentam detritos produzidos durante o processo construtivo, reforma ou demolição de edifícios, estradas, pontes, dentre as diversas atividades relacionados à engenharia civil (UNITED STATES ENVIRONMENTAL PROTECTION AGENCY, 2016), e geralmente são constituídos de grande volume e massa, dentre estes o concreto, madeira, asfalto, gesso (o principal componente de

Revista Produção Online. Florianópolis, SC, v. 20, n. 2, p. 449-471, 2020 
drywall), metais, tijolos, vidro, plásticos, PVC, solo e rochas (ESA; HALOG; RIGAMONTI, 2016).

Apenas de $20 \%$ a $30 \%$ dos resíduos provenientes da construção e demolição de edifícios são reutilizados ou reciclados, sendo que tal setor possui muitos materiais reutilizáveis como: concreto, madeira e aço (WOLRD ECONOMIC FORUM, 2014). Os RCD são gerados por diversos motivos, sendo os principais o excesso de pedidos, o design, e as falhas no planejamento (SILVA; VITHANA, 2008).

Todas as atividades no ambiente construtivo geram desperdício, umas mais que outras, podendo vir de compras excessivas ou da personalização de materiais para se adequarem à arquitetura da obra (COCHRAN; TOWNSEND, 2010).

As práticas propostas pelos fundamentos da EC são amplamente aplicáveis em grandes, médios e pequenos empreendimentos. Porém, ainda há muita resistência em sua efetiva aplicação, principalmente em pequenas empresas e edificações. Existem barreiras estruturais, contextuais e culturais que são desencadeadas pela flexibilidade da economia, estrutura organizacional e falta de incentivos orçamentários (LIU; BAI, 2014).

Para Sauvé, Bernard e Sloan (2016, p. 52) a EC objetiva "otimizar o uso de recursos, reduzir a poluição e o desperdício em cada etapa, na medida do possível e desejável". Para Pomponi e Moncaster (2017), a principal inovação dentro da ideia de uma EC consiste em desassociar o esgotamento e o crescimento do consumo de recursos, permitindo assim que haja desenvolvimento econômico e rentabilidade cada vez maiores, sem que exista uma pressão crescente sobre o meio ambiente.

Para Sauvé, Bernard e Sloan (2016, p. 49) a EC é conceituada como um modelo de "produção e consumo de bens através de fluxos de materiais de circuito fechado que analisam externalidades ambientais ligadas à extração de recursos virgens e à geração de resíduos", inclusive a poluição. Já Murray, Skene e Haynes (2015, p. 377) oferecem uma definição revisada da EC, em que esta é considerada como um modelo econômico onde "planejamento, recursos, compras, produção e reprocessamento são projetados e gerenciados, tanto como processo como em resultados, para maximizar o funcionamento do ecossistema e o bem-estar humano". 
Andersen (2007) afirma que a EC aborda as interligações das quatro funções econômicas do meio ambiente: ser um sistema fundamental de suporte à vida, proporcionar qualidade de vida, ser base para o fornecimento de recursos e receber os descartes de materiais provenientes das atividades econômicas. Já Geissdoerfer et al. (2017) afirmam que a literatura demonstra que o sistema priorizado na sustentabilidade é o triple bottom line, já na EC o sistema econômico é percebido como mais relevante.

De acordo com Ellen MacArthur Foundation (2013, p. 14), a EC ainda é uma construção teórica, sendo que o termo "denota uma economia industrial que é restauradora ou regenerativa por intenção e por design", e que vem ganhando força desde o final da década de 1970. McDonough et al. (2003) classificam a EC como um sistema que se alimenta de energia renovável, onde os materiais avançam em circuitos fechados, seguros e regenerativos.

Percebe-se que os conceitos apresentados sobre economia circular têm em comum o potencial de reduzir os impactos ambientais causados pelas atividades ambientais e que está em acordo com os fundamentos da sustentabilidade. 0 Quadro 1 apresenta, resumidamente, a compreensão dos principais autores sobre economia circular.

Quadro 1 - Síntese do conceito de Economia Circular

\begin{tabular}{|l|l|}
\hline \multicolumn{1}{|c|}{ Autoria } & \multicolumn{1}{c|}{ Conceito de Economia Circular } \\
\hline $\begin{array}{l}\text { ELLEN MACARTHUR } \\
\text { FOUNDATION (2013) }\end{array}$ & $\begin{array}{l}\text { Uma nova maneira de gerir os resíduos, de forma que estes sejam } \\
\text { desenvolvidos e melhorados para o retorno à cadeia produtiva. }\end{array}$ \\
\hline $\begin{array}{l}\text { SAUVÉ; BERNARD; SLOAN } \\
\text { (2016). }\end{array}$ & $\begin{array}{l}\text { Seus fundamentos direcionam a sociedade a otimizar e reduzir o } \\
\text { uso dos recursos naturais, reduzindo assim o desperdício e a } \\
\text { poluição. }\end{array}$ \\
\hline $\begin{array}{l}\text { FOSTER, ROBERTO, IGARI } \\
\text { (2016). }\end{array}$ & $\begin{array}{l}\text { Metodologia que exclui a inutilização de rejeitos em seu ciclo } \\
\text { produtivo. }\end{array}$ \\
\hline $\begin{array}{l}\text { POMPONI; MONCASTER } \\
\text { (2017) }\end{array}$ & $\begin{array}{l}\text { Modelo produtivo que põe fim ao esgotamento e ao crescimento do } \\
\text { consumo de recursos. }\end{array}$ \\
\hline MCDONOUGH et al., (2003). & $\begin{array}{l}\text { Sistema no qual se utiliza energia renovável e seus materiais, } \\
\text { dentro de sua cadeia produtiva, que participam de um ciclo } \\
\text { fechado, seguro e regenerativo. }\end{array}$ \\
\hline
\end{tabular}

Fonte: Autores (2018)

\section{METODOLOGIA}

A metodologia aplicada a essa pesquisa foi o estudo de caso, pois é uma investigação sobre a compreensão da dinâmica presente em uma configuração única e tipicamente combina a coleta de dados, de arquivos, entrevistas,

Revista Produção Online. Florianópolis, SC, v. 20, n. 2, p. 449-471, 2020 
questionários e a observação in loco. Os estudos de casos são considerados adequados quando a estratégia de pesquisa é estudar o fenômeno empiricamente em seu contexto (EINSENHARDT, 1989).

As evidências coletadas nos estudos de casos podem ser qualitativas, quantitativas ou ambas. Os dados qualitativos são úteis para entender a teoria e relacionar os dados quantitativos, enquanto os dados quantitativos podem indicar relacionamentos que não podem ser salientados na pesquisa qualitativa (EINSENHARDT, 1989; YIN, 2010).

As questões do roteiro de entrevista foram norteadas pela literatura e objetivo do trabalho. O roteiro de entrevista foi composto por 12 questões abertas, divididas em três seções: a primeira busca identificar a empresa estudada, o portfólio de produtos, serviços e missão; a segunda contempla questões sobre consumidores, afim de identificar ações de EC e sustentabilidade; a terceira apresenta questões sobre o impacto das atividades das empresas no meio ambiente, apresentando uma régua de 0 a 10 pontos ( 0 - menor importância e 10 - maior importância). Essa métrica foi aplicada quando os entrevistados eram convidados a identificar qual o posicionamento da empresa em relação ao seu grau de preocupação com os produtos fabricados e o impacto no meio ambiente em uma escala de 0 até 10 .

Além das entrevistas, documentos, arquivos e outros materiais, todos fornecidos pelas empresas, puderam ser analisadas informações secundárias (sites, relatórios, reportagens), de modo a complementar o estudo. Destaca-se que os estudos de caso foram realizados em empresas que se enquadram nos princípios da EC e que estão localizadas na Região Metropolitana do Cariri-CE.

É válido ressaltar que o conjunto urbano da Região Metropolitana do Cariri (RMC) é a segunda região urbana mais expressiva do Ceará. É formada por nove municípios, onde possui três cidades principais (Juazeiro do Norte, Crato e Barbalha, conhecidas também por CRAJUBAR). Ao criar a RMC, o Governo do Estado do Ceará objetivou a concepção de um polo de desenvolvimento socioeconômico que pudesse atrair investimentos ao interior do estado (GOVERNO DO ESTADO DO CEARÁ, 2020). Por essa razão, a conurbação dos municípios do triângulo CRAJUBAR movimenta o comércio local e atrai investimentos de empresas que fornecem insumos e materiais para toda a região. 
Os dois estudos de caso foram selecionados de acordo com o conhecimento dos autores e divulgação das atividades das empresas na mídia regional. Um total de 5 empresas do setor da construção civil foram contatadas, porém apenas duas aceitaram participar da pesquisa, conforme Quadro 2.

Quadro 2 - Seleção dos casos

\begin{tabular}{|c|l|l|}
\hline Casos & \multicolumn{1}{|c|}{ Empresa Privada 1 } & \multicolumn{1}{c|}{ Empresa Privada 2 } \\
\hline Foco do estudo & $\begin{array}{l}\text { Fabricação e distribuição de } \\
\text { gesso acartonado }\end{array}$ & $\begin{array}{l}\text { Fabricação e instalação de esquadrias de } \\
\text { alumínio }\end{array}$ \\
\hline Localização & Juazeiro do Norte-CE & Filial Juazeiro do Norte-CE \\
\hline Entrevistado & Gerente de produção & Gerente de produção \\
\hline
\end{tabular}

Fonte: Autores (2018).

As entrevistas foram realizadas no período de outubro de 2017 a fevereiro de 2018, com a feitura de duas entrevistas em cada empresa, além da realização de análise de dados secundários.

As entrevistas foram gravadas e transcritas com duração média de duas horas e, em seguida, as informações foram apresentadas aos entrevistados para validação. Os nomes das empresas foram preservados.

Para representar o fluxo dos materiais das empresas foi utilizado o software Bizagi e as informações foram validadas pelos entrevistados.

\section{RESULTADOS E DISCUSSÃO}

\subsection{Empresa 1: Fabricação e distribuição de gesso acartonado}

A empresa estudada faz parte de uma das mais reconhecidas no Brasil no que diz respeito ao fornecimento de matérias-primas para a construção a seco. Além disso, é a única cuja fabricação de drywall é inteiramente em território nacional. Ela desenvolve suas atividades desde 2008 na cidade de Juazeiro do Norte-CE, e já é reconhecida pelo seu fornecimento de qualidade na produção de chapas de drywall e de massa pronta para o tratamento de juntas e acabamento. Além disso, faz parte do Green Building Council Brazil, reconhecida pela adoção de práticas de construção verde, oferecendo soluções e serviços eficientes do ponto de vista ambiental. 
O drywall é uma solução viável para as obras tanto do ponto de vista econômico quanto ambiental, uma vez que leva menos tempo em sua instalação, traz vantagens no transporte manual, já que é leve e gera menos entulho (ASSOCIAÇÃO DO DRYWALL, 2015), sendo assim uma opção para substituir as vedações internas da edificação de alvenaria convencional.

A partir da entrevista, percebeu-se que a empresa conta com uma abordagem sistêmica e inovadora no que diz respeito a produção de drywall, além de estar atenta aos diversos fatores que têm influência no fluxo reverso da produção e de possuir rigorosos padrões de qualidade. Ademais, o público tem conhecimento detalhado da fabricação, dos benefícios e vantagens do produto através do departamento de marketing e técnico, e contam com uma central de reclamações sobre a qualidade.

O mercado consumidor da empresa abrange todo o território nacional, pois conta com mais de 50 distribuidores e instaladores, desde pequenas até grandes construtoras. Entretanto, com base em informações obtidas no site da empresa, percebe-se que ela ainda encontra resistência na venda de sua mercadoria em construções de pequeno porte e residências, devido à falta de conhecimento dos construtores.

O entrevistado 1 informou que:

"[...] boa parte das perdas do drywall no processo de fabricação são reprocessados, o que acarretou uma considerável diminuição na quantidade de resíduos gerados. Porém, essa reutilização está restrita apenas aos resíduos secos".

O processo tradicional de produção das placas de gesso acartonado, conforme a Figura 3, parte da fabricação de gesso, quando este é transportado por meio de roscas até um equipamento denominado "misturador". Em seguida o gesso é misturado com água e aditivos, produzindo uma chapa contínua, que posteriormente é cortada e encaminhada a um equipamento denominado "secador" que, por sua vez, possui a função de retirar toda a umidade da chapa fabricada. Após o processo de secagem, as chapas passam por acabamento, quando são colocadas em esquadros e posteriormente paletizadas e embaladas para ser encaminhadas ao estoque.

O conceito de EC é amplamente aplicado quando utilizam os rejeitos de gesso tanto das obras quanto do próprio processo de fabricação, conforme as Revista Produção Online. Florianópolis, SC, v. 20, n. 2, p. 449-471, 2020 
atividades do campo "Reaproveitamento" da Figura 3. Os rejeitos são aprimorados de forma que consigam retornar à produção, assim como previsto por Ellen Macarthur Foundation (2013).

A empresa analisada recolhe esses rejeitos e, de acordo com o entrevistado:

"[...] 3\% dos resíduos são separados e destinados a uma forrageira que realiza um processo de trituração, em seguida encaminhados para peneiras. No processo de peneiras é feita a separação do papel cartão e do gesso processado, este gesso é inserido no processo de fabricação da chapa como aditivo seco chamado de acelerante" - Entrevistado 1.

A empresa reconhece que as chapas de gesso acartonado são produtos $100 \%$ recicláveis e que no processo de fabricação do produto o consumo de energia por $\mathrm{m}^{2}$ é menor. Além disso, os consumidores têm a vantagem de contar com o orçamento prévio mais preciso, os custos com a fundação das obras também são diminuídos e o processo construtivo acaba sendo mais leve, tanto no peso total da construção quanto no peso que os operários transportam, benefícios estes já observados pela Associação do Drywall (2015).

\subsection{Empresa 2: Fabricação e instalação de esquadrias de alumínio}

A empresa atua no mercado caririense da construção civil desde a década de 1990, na produção e instalação de esquadrias de alumínio, fachadas unitizadas e revestimentos em alumínio composto. Além disso, integra a Associação Nacional de Fabricantes de Esquadrias de Alumínio (AFEAL).

No que compreende à aplicação dos conceitos de EC, de acordo com o entrevistado, a empresa trabalha com a remanufatura de resíduos de alumínio para confecção de artesanato, direcionando-os para artesãos localizados no estado da Bahia. Com isso, busca-se reduzir o descarte das sobras de alumínio oriundas dos serviços fornecidos pela empresa. Tais resíduos são transferidos para suas instalações e reinseridas na cadeia de suprimentos, gerando assim lucros para comunidades baianas, promovendo desenvolvimento sustentável.

O cliente conhece os detalhes de fabricação e os testes de desempenho do produto através de memorial de cálculo e por meio de propaganda feita pelo departamento responsável. A organização atende a grandes e pequenas construtoras e preza pela qualidade de seus produtos, tanto que detém a 
certificação da Qualidade ISO 9001. Líder na região Nordeste no segmento de fachadas pele de vidro e revestimento em alumínio composto, a empresa atua nos estados de Pernambuco, Bahia, Sergipe, Alagoas, Paraíba, Rio Grande do Norte, Ceará, Maranhão e Pará.

Durante a entrevista, foi solicitado ao entrevistado o detalhamento do procedimento de produção a que os remanufaturados são submetidos, quando este informou que o material é direcionado à fábrica em barra ou chapa, onde são usinados e, posteriormente, cortados, de acordo com as especificações do projeto, conforme Figura 4.

O entrevistado foi questionado se conhecia o conceito de EC, no que respondeu:

"[...] conheço o conceito, mas não em sua totalidade, apesar de aplicar na prática seus conceitos, para a empresa as atividades de reutilização dos resíduos do alumínio estão relacionadas à diminuição do impacto ambiental $e$ não associam ao conceito de economia circular, por falta de conhecimento." - Entrevistado 2.

Percebe-se que o alumínio tem importante participação no processo de transição da Economia Linear para a Economia Circular. Segundo a Associação do Alumínio (2017), o Brasil é o $11^{\circ}$ produtor de alumínio primário, precedido por China, Rússia, Canadá, Emirados Árabes, Índia, Austrália, Noruega, Bahrein e Estados Unidos. Nesse contexto, a iniciativa dessa organização merece reconhecimento no tocante ao desenvolvimento de suas atividades, pois o alumínio é um material propício à reutilização, sem que haja a perda de suas propriedades de qualidade.

De acordo com as empresas analisadas, os clientes adquirem os seus produtos por fatores diversos e não relacionados ao processo de EC e/ou preocupação da empresa com o meio ambiente, conforme Figura 5. Os entrevistados foram unânimes em afirmar que os clientes adquirem seus produtos por reconhecerem a qualidade e os benefícios dos produtos ofertados.

Apenas o entrevistado 1 afirmou que os produtos são adquiridos porque os clientes reconhecem que os produtos são voltados ao processo de EC e/ou desenvolvimento sustentável.

Quando questionados quanto a preocupação com o impacto ambiental gerado por suas atividades, solicitou-se que os entrevistados avaliassem tal questão e colocassem em uma escala de 0 a 10 (considerando 0 como menos importante e 10 como mais importante). As respostas foram apresentadas na Figura 6.

Revista Produção Online. Florianópolis, SC, v. 20, n. 2, p. 449-471, 2020 
Assim, quando questionados sobre o grau de preocupação das atividades da empresa com o impacto no meio ambiente, os entrevistados indicaram dar alta importância ao meio ambiente, bem como demonstraram se preocupar com a mitigação do impacto ao meio ambiente. Isso é notado pelos produtos e atividades que desempenham e também é uma preocupação da economia circular. 
Figura 3 - Processos de produção do Drywall

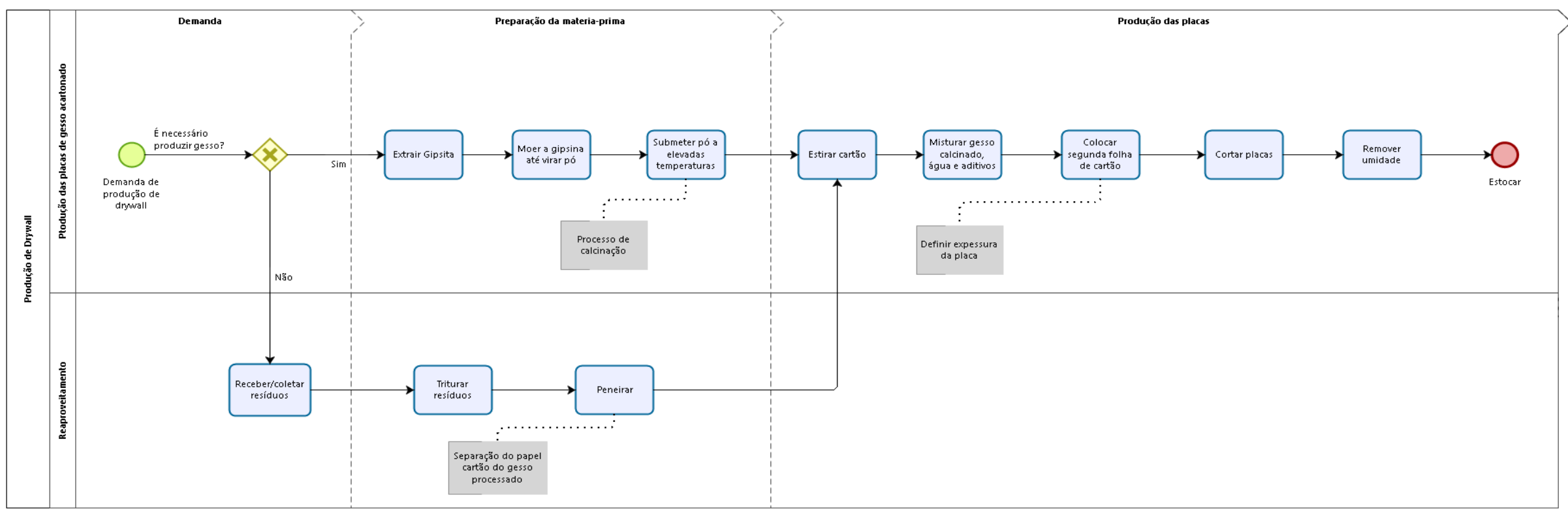

Fonte: Autores (2018). 
Figura 4 - Processos de produção das chapas de alumínio

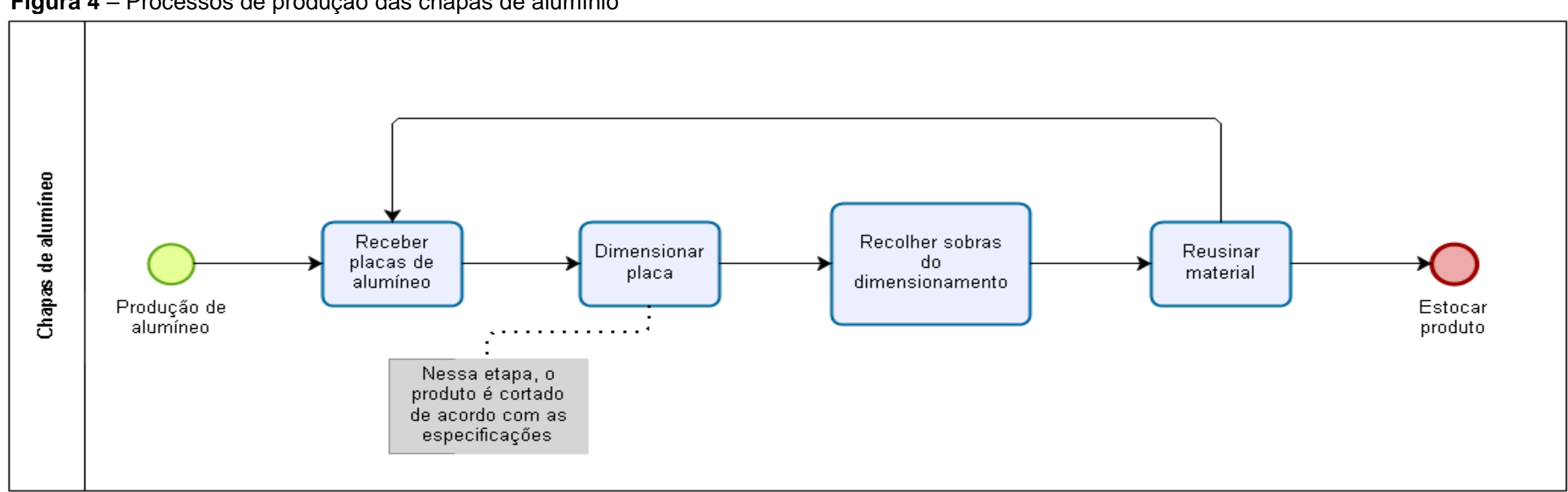

Fonte: Autores (2018) 
Figura 5 - Motivos para aquisição dos produtos

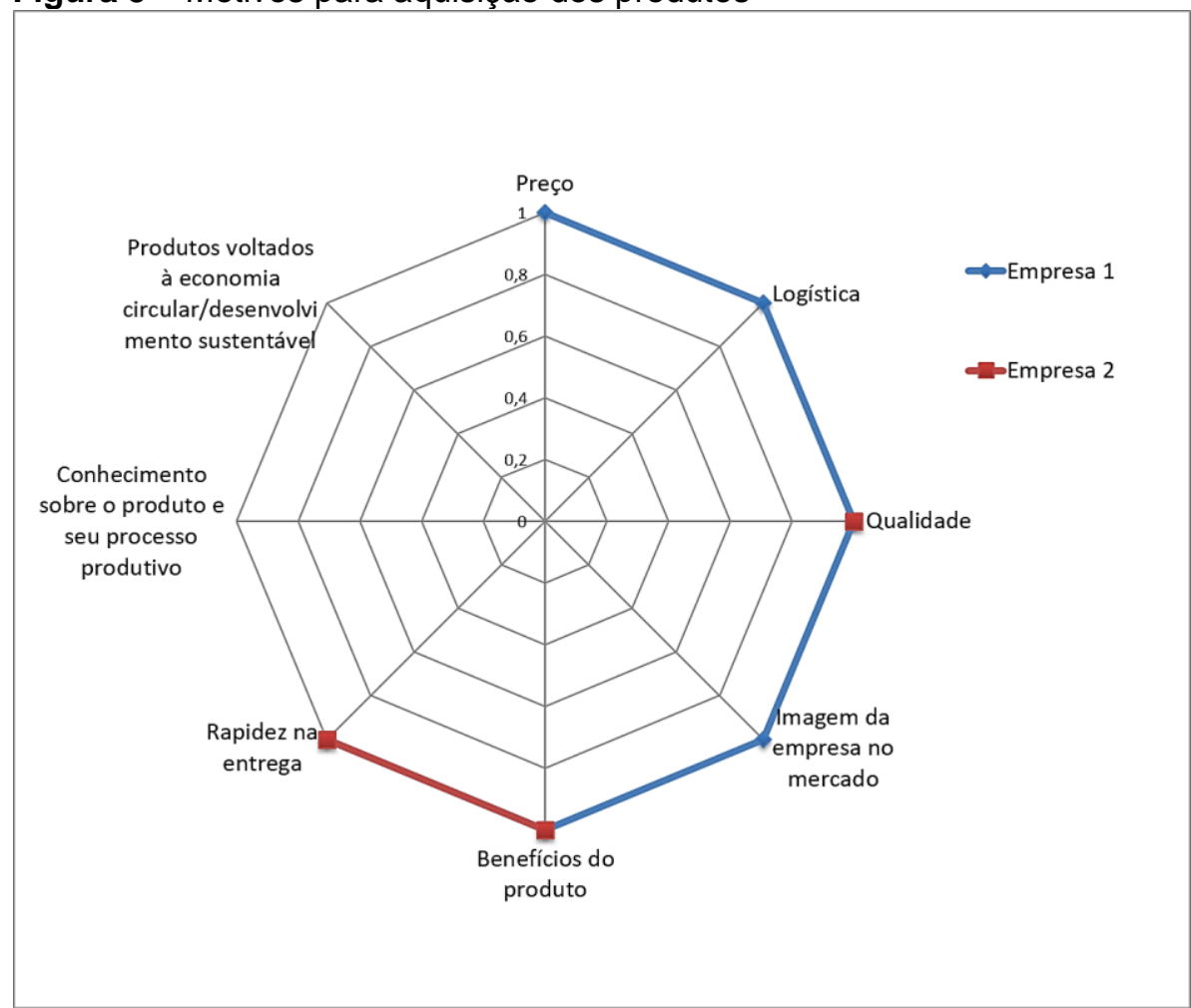

Fonte: Autores (2018).

Figura 6 - Grau de preocupação dos produtos fabricados com o impacto no meio ambiente Menor Importância

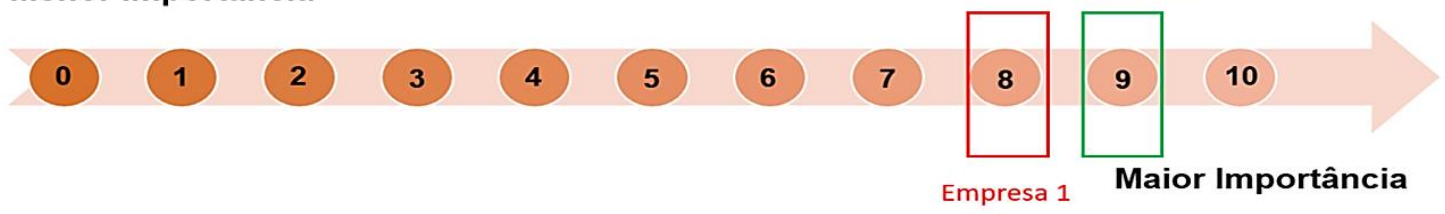

Fonte: Autores (2018).

De acordo com Araújo e Queiroz (2017), a EC pode ser considerada um ciclo de desenvolvimento contínuo que preserva e aprimora o capital natural, otimizando a produção de recursos, minimizando riscos sistêmicos, administrando estoques finitos e fluxos renováveis, oferecendo diversos mecanismos de criação de valor dissociados do consumo de recursos finitos.

Analisando as duas empresas estudadas, do ponto de vista da reutilização dos resíduos, pode-se concluir que elas desenvolvem ações baseadas no conceito da EC. De acordo com Ellen Macarthur Foundation (2013), a EC é uma nova maneira de gerir os resíduos, de forma que estes são desenvolvidos e melhorados para o retorno à cadeia produtiva. Ainda, segundo Sauvé, Bernard e Sloan (2016), fundamentos da EC direcionam a sociedade a otimizar e reduzir o uso dos recursos Revista Produção Online. Florianópolis, SC, v. 20, n. 2, p. 449-471, 2020 
naturais, reduzindo assim o desperdício e a poluição. Sob a perspectiva desses autores, verifica-se que as atividades das empresas estão alinhadas ao conceito de EC.

Entretanto, no caso 2 é necessário ter mais evidências, bem como, analisar outros atores da cadeia, visando verificar se a renda gerada para as comunidades locais com o uso dos resíduos para artesanato, é justa. Outro fator a se considerar é que, em ambos os casos, nota-se que as empresas estão preocupadas com os resíduos e sua destinação. Outro ponto abordado pela EC é a redução no consumo de matéria-prima para que a quantidade de resíduos também seja mitigada, quesito este que não foi mencionado pelos entrevistados. Quando questionados, os participantes informaram que se preocupam apenas com os resíduos e sua mitigação ou em dar um destino correto a estas sobras. Além disso, a EC gera benefícios como a oportunidade de crescimento econômico e de inovação para a empresa, geração de emprego, diminuição da importação de produtos e redução dos impactos ambientais, de acordo com Simões, Almeida e Santos (2017). Entretanto, as empresas estudadas destacaram apenas os benefícios de redução dos impactos ambientais, não mensurando outras variáveis. Assim, as ações estão mais voltadas ao desenvolvimento sustentável.

\section{CONSIDERAÇÕES FINAIS}

Este trabalho teve por objetivo analisar os princípios da Economia Circular no setor da construção civil cearense. O método de pesquisa selecionado foi o de estudos de caso realizados em duas empresas do setor da construção civil cearense.

A análise da literatura demonstrou que os conceitos de EC e de Sustentabilidade têm comumente o objetivo de propor uma reorganização dos meios de exploração das matérias-primas, que são limitadas, de maneira a favorecer à economia, ao meio ambiente e a sociedade. Aplicando ambas as definições na construção civil, pode-se obter menos impactos ambientais em suas atividades, sejam elas na construção, demolição ou reforma, não importando o porte da obra.

A transição da EL para EC revela-se como uma alternativa eficaz para a mudança da sistematização dos fluxos dos resíduos sólidos da construção civil, uma vez que há o reaproveitamento de materiais tanto dentro, quanto fora de sua cadeia Revista Produção Online. Florianópolis, SC, v. 20, n. 2, p. 449-471, 2020 
produtiva original, além de respeitar os limites ambientais e favorecer economicamente quem a aplica em sua organização.

Entretanto, para a maximização do lucro a partir da EC, percebe-se que as empresas necessitam de um bom planejamento logístico reverso com um grau maior de complexidade pois os resíduos gerados necessitam retornar as fábricas para serem reprocessados, como visto no Estudo de Caso 1. Segundo Stocher (2019) há atração de clientes por organizações que dispõem sistema de coleta visível pois esta cria a ideia de responsabilidade sustentável. Porém, se o reuso dos materiais for feito dentro das próprias empresas é necessário apenas um pequeno planejamento da disposição destes materiais no layout disponível, de modo que não atrapalhe o fluxo produtivo, como acontece no Caso 2.

A implementação dos conceitos de EC no ambiente construtivo do cariri cearense demandará bastante tempo já que tanto os empreendedores quanto os clientes apresentam certa resistência a novas metodologias, apesar de que os estudos de caso apresentaram um grau elevado de preocupação com o meio ambiente. Percebeu-se, também, que o consumidor final tem papel decisivo no incentivo de aplicação da EC, visto que se os consumidores adquirissem mais produtos sustentáveis, as empresas investiriam mais na reutilização dos materiais e o usariam para beneficiamento próprio através do marketing. As limitações deste estudo estão na impossibilidade de generalizar os dados obtidos devido ao tamanho da amostra e ao método escolhido. Futuras pesquisas podem aumentar as quantidades amostradas, selecionando os materiais mais utilizados na construção civil e realizando um estudo sobre o destino desses resíduos/rejeitos, com o intuito de verificar a existência de um processo de Economia Circular. Além disso, pode-se analisar os fatores sistêmicos que influenciam a reestruturação do modelo produtivo atual, o linear, e os obstáculos encontrados nesse processo na região do Cariri cearense.

Este estudo contribui com os gestores para que compreendam o conceito de Economia Circular e sua importância para a construção civil, minimizando a quantidade de resíduos/rejeitos que este setor dispõe ao meio ambiente.

\section{REFERÊNCIAS}

ABRELP. Panorama de resíduos sólidos no Brasil 2016. Disponível em:

Revista Produção Online. Florianópolis, SC, v. 20, n. 2, p. 449-471, 2020 
http://www.mpdft.mp.br/portal/pdf/comunicacao/junho 2018/panoramaanexos2016.pdf. Acesso em: 04 nov. 2018.

ANDERSEN, Mikael Skou. An introductory note on the environmental economics of the circular economy. Sustainability Science, [s.I.], v. 2, n. 1, p. 133-140, 21 dez. 2006. http://dx.doi.org/10.1007/s11625-006-0013-6.

ARAÚJO, T.D.; QUEIROZ, A.A.F.S.L. Economia circular: breve panorama da produção científica entre 2007 e 2017. In: ENCONTRO INTERNACIONAL SOBRE GESTÃO AMBIENTAL E MEIO AMBIENTE (ENGEMA), 19., 2017. [Anais...].,São Paulo: FEA/USP:, 2017. p. 1-17. Disponível em:

http://engemausp.submissao.com.br/19/anais/arquivos/417.pdf. Acesso em: 07 nov. 2018.

ARUP (London). The circular economy in the built environment. 2016. Disponível em: https://www.arup.com/-/media/arup/files/publications/c/circular-economy-in-the-builtenvironment-270916.pdf. Acesso em: 24 fev. 2018.

ASSOCIAÇÃO BRASILEIRA DO ALUMÍNIO (São Paulo). Perfil da indústria brasileira do alumínio. 2017. Disponível em: http://abal.org.br/estatisticas/nacionais/perfil-da-industria/. Acesso em: 24 fev. 2018.

ASSOCIAÇÃO BRASILEIRA DOS FABRICANTES DE CHAPAS PARA DRYWALL. Saiba mais sobre o drywall e suas vantagens. São Paulo. Disponível em:

http://www.drywall.org.br/imprensa.php/1/967/saiba-mais-sobre-o-drywall-e-conheca-suasvantagens. Acesso em: 24 fev. 2018.

ASSOCIAÇÃO DO DRYWALL. Resíduos de gesso na construção civil. 2015. Disponível em: http://www.drywall.org.br/biblioteca.php/1/3/dl/44/residuos-de-gesso-na-construcao-civil. Acesso em: 4 nov. 2018.

BIDONE, F. A. Resíduos sólidos provenientes de coletas especiais: eliminação e valorização. Brasília: FINEP/PROSAB, 2001.

BRASIL. INSTITUTO BRASILEIRO DE GEOGRAFIA E ESTATÍSTICA. Pesquisa industrial mensal de emprego e salário. 2017. Disponível em:

https://ww2.ibge.gov.br/home/estatistica/indicadores/industria/pimes/default.shtm. Acesso em: 4 nov. 2018.

CHERTOW, Marian R.. Industrialsymbiosis: literature and taxonomy. Annual Review Of Energy And The Environment, [s.I.], v. 25, n. 1, p. 313-337, nov. 2000. http://dx.doi.org/10.1146/annurev.energy.25.1.313.

COCHRAN, K.m.; TOWNSEND, T.g.. Estimating construction and demolition debris generation using a materials flow analysis approach. Waste Management, [s.I.], v. 30, n. 11, p. 2247-2254, nov. 2010. http://dx.doi.org/10.1016/j.wasman.2010.04.008.

DUAN, Huabo; LI, Jinhui. Construction and demolition waste management: china's lessons. Waste Management \& Research, [s.I.], v. 34, n. 5, p. 397-398, maio 2016. http://dx.doi.org/10.1177/0734242x16647603.

EISENHARDT, Kathleen M.. Building Theories from Case Study Research. The Academy Of Management Review, [s.I.], v. 14, n. 4, p. 532-891, out. 1989.

http://dx.doi.org/10.2307/258557. 
ELLEN MACARTHUR FOUNDATION (EMF). Rumo à economia circular: o racional de negócio para acelerar a transição, 2015. Disponível em:

https://www.ellenmacarthurfoundation.org/assets/downloads/Rumo-\%C3\%A0-economiacircular SumarioExecutivo.pdf. Acesso em: 23 fev. 2018.

ELLEN MACARTHUR FOUNDATION (EMF). Towards the Circular Economy, v. 1, 2013. Disponível em:

https://www.ellenmacarthurfoundation.org/assets/downloads/publications/Ellen-MacArthurFoundation-Towards-the-Circular-Economy-vol.1.pdf . Acesso em: 23 fev. 2018.

ELLEN MACARTHUR FOUNDATION (EMF). Uma economia circular no Brasil: uma abordagem exploratória inicial, 2017. Disponível em https://www.ellenmacarthurfoundation.org/assets/downloads/languages/Uma-EconomiaCircular-no-Brasil Uma-Exploracao-Inicial.pdf. Acesso em: 24 fev. 2018.

ESA, Mohd Reza; HALOG, Anthony; RIGAMONTI, Lucia. Developing strategies for managing construction and demolition wastes in Malaysia based on the concept of circular economy. Journal Of Material Cycles And Waste Management, [s.I.], v. 19, n. 3, p. 11441154, 1 jun. 2016. http://dx.doi.org/10.1007/s10163-016-0516-x.

FOSTER, A.; ROBERTO, S. S.; IGARI, A. T. Economia circular e resíduos sólidos: uma revisão sistemática sobre a eficiência ambiental e econômica, 2016.

FLORIM, Leila Chagas; QUELHAS, Osvaldo Luiz. Contribuição para a construção sustentável: características de um projeto habitacional eco-eficiente. Revista Produção Online, [s.I.], v. 5, n. 2, p. 327-345, 24 jun. 2005. http://dx.doi.org/10.14488/16761901.v5i2.332.

GEISSDOERFER, Martin; SAVAGET, Paulo; BOCKEN, Nancy M.p.; HULTINK, Erik Jan. The circular economy: a new sustainability paradigm? Journal Of Cleaner Production, [s.I.], v. 143, p. 757-768, fev. 2017. http://dx.doi.org/10.1016/.j.jelepro.2016.12.048.

GENG, Yong; DOBERSTEIN, Brent. Developing the circular economy in China: challenges and opportunities for achieving 'leapfrog development. International Journal Of Sustainable Development \& World Ecology, [s.I.], v. 15, n. 3, p. 231-239, jun. 2008. http://dx.doi.org/10.3843/susdev.15.3:6.

GENG, Yong; FUJITA, Tsuyoshi; PARK, Hung-suck; CHIU, Anthony; HUISINGH, Donald. Call for papers: towards post fossil carbon societies. Journal Of Cleaner Production, [s.I.], v. 68, p. 4-6, abr. 2014. http://dx.doi.org/10.1016/j.jclepro.2013.12.089.

GHISELLINI, Patrizia; CIALANI, Catia; ULGIATI, Sergio. A review on circular economy: the expected transition to a balanced interplay of environmental and economic systems. Journal Of Cleaner Production, [s.I.], v. 114, p. 11-32, fev. 2016.

http://dx.doi.org/10.1016/j.jclepro.2015.09.007.

GOVERNO DO ESTADO DO CEARÁ. Região Metropolitana do Cariri. 2020. Disponível em: https://www.cidades.ce.gov.br/regiao-metropolitana-do-cariri/. Acesso em: 07 maio 2020 .

HILSDORF, Wilson de Castro; LOPES, Ana Paula Vilas Boas Viveiros; CITTATINI, Caroline; GHISINI, Juliana Siqueira. Aplicação de ferramentas do lean manufacturing: estudo de caso em uma indústria de remanufatura. Revista Produção Online, [s.I.], v. 19, n. 2, p. 640-667, 15 jun. 2019. http://dx.doi.org/10.14488/1676-1901.v19i2.3391.

Revista Produção Online. Florianópolis, SC, v. 20, n. 2, p. 449-471, 2020 
HOLDEN, Erling; LINNERUD, Kristin; BANISTER, David. The Imperatives of Sustainable Development. Sustainable Development, [s.I.], v. 25, n. 3, p. 213-226, 30 set. 2016. http://dx.doi.org/10.1002/sd.1647.

JUNIOR, Joel Vieira; ROMANEL, Celso. Sustentabilidade na indústria da construção: uma logística para reciclagem dos resíduos de pequenas obras. Urbe - Revista Brasileira de Gestão Urbana, [s.I.], v. 5, n. 480, p. 27-41, 2013.

http://dx.doi.org/10.7213/urbe.05.002.se02.

LETT, Lina A.. Las amenazas globales, el reciclaje de residuos y el concepto de economía circular. Revista Argentina de Microbiología, [s.I.], v. 46, n. 1, p. 1-2, jan. 2014. http://dx.doi.org/10.1016/s0325-7541(14)70039-2.

LIU, Yong; BAI, Yin. An exploration of firms' awareness and behavior of developing circular economy: an empirical research in china. Resources, Conservation And Recycling, [s.I.], v. 87, p. 145-152, jun. 2014. http://dx.doi.org/10.1016/j.resconrec.2014.04.002.

MATHEWS, John A.; TAN, Hao. Progress toward a circular economy in China. Journal Of Industrial Ecology, [s.I.], v. 15, n. 3, p. 435-457, 30 mar. 2011.

http://dx.doi.org/10.1111/j.1530-9290.2011.00332.x.

MCDONOUGH, William; BRAUNGART, Michael; ANASTAS, Paul T.; ZIMMERMAN, Julie B. Peer Reviewed: applying the principles of green engineering to cradle-to-cradle design:

Environmental Science \& Technology, [s.I.], v. 37, n. 23, p. 434-441, dez. 2003. http://dx.doi.org/10.1021/es0326322.

MURRAY, Alan; SKENE, Keith; HAYNES, Kathryn. The Circular Economy: an interdisciplinary exploration of the concept and application in a global context. Journal Of Business Ethics, [s.I.], v. 140, n. 3, p. 369-380, 22 maio 2015.

http://dx.doi.org/10.1007/s10551-015-2693-2.

NAUSTDALSLID, Jon. Circular economy in China - the environmental dimension of the harmonious society. International Journal Of Sustainable Development \& World Ecology, [s.l.], v. 21, n. 4, p. 303-313, 16 maio 2014. http://dx.doi.org/10.1080/13504509.2014.914599.

NESS, David. Sustainable urban infrastructure in China: Towards a Factor 10 improvement in resource productivity through integrated infrastructure systems. The International Journal Of Sustainable Development And World Ecology, v. 15, n. 8, p. 23-40, jan. 2008.

NETO, J. C. M. Gestão dos resíduos de construção e demolição no Brasil. RiMa, 2005.

NOYA, Isabel; ALDEA, Xavier; GONZÁLEZ-GARCÍA, Sara; GASOL, Carles M.; MOREIRA, María Teresa; AMORES, Maria José; MARÍN, Desirée; BOSCHMONART-RIVES, Jesús. Environmental assessment of the entire pork value chain in Catalonia: a strategy to work towards circular economy. Science Of The Total Environment, [s.I.], v. 589, p. 122-129, jul. 2017. http://dx.doi.org/10.1016/..scitotenv.2017.02.186.

POMPONI, Francesco; MONCASTER, Alice. Circular economy for the built environment: a research framework. : A research framework. Journal Of Cleaner Production, [s.I.], v. 143, p. 710-718, fev. 2017. http://dx.doi.org/10.1016/j.jclepro.2016.12.055. 
SANCHEZ, Benjamin; HAAS, Carl. Capital project planning for a circular economy. Construction Management And Economics, [s.I.], v. 36, n. 6, p. 303-312, 15 fev. 2018. http://dx.doi.org/10.1080/01446193.2018.1435895.

SAUVÉ, Sébastien; BERNARD, Sophie; SLOAN, Pamela. Environmental sciences, sustainable development and circular economy: alternative concepts for trans-disciplinary research. Environmental Development, [s.I.], v. 17, p. 48-56, jan. 2016. http://dx.doi.org/10.1016/..envdev.2015.09.002.

SILVA, Nayanthara de; VITHANA, S.b.k.h.. Use of PC elements for waste minimization in the Sri Lankan construction industry. Structural Survey, [s.I.], v. 26, n. 3, p. 188-198, 11 jul. 2008. http://dx.doi.org/10.1108/02630800810887081.

SIMÕES, A.; ALMEIDA, M.; SANTOS, D. Economia Circular na Indústria Cerâmica: Proposta de classificação do resíduo "caco cozido" como subproduto. 2017. 74 f. Relatório de Estágio Profissionalizante (Mestrado em Gestão Ambiental)- Escola Superior Agrária de Coimbra, Coimbra, 2017. 1. Disponível em: https://comum.rcaap.pt/bitstream/10400.26/20925/1/Relat\%C3\%B3rio Est\%C3\%A1gioMGA-Filipa\%20Sim\%C3\%B5es.pdf. Acesso em: 04 nov. 2018.

STAHEL, W. Product-life factor. Mitchell Prize Winning Paper, 1982. Disponível em: http://www.product-life.org/en/major-publications/the-product-life-factor. Acesso em: 4 nov. 2018.

STOCHER, Francielli Moreira; SILVA, Mygre Lopes da; CAPPELLARI, Gabriela; CASSANEGO JUNIOR, Paulo Vanderlei. A logística reversa no setor farmacêutico. Revista Produção Online, [s.l.], v. 19, n. 3, p. 1069-1093, 16 set. 2019.

http://dx.doi.org/10.14488/1676-1901.v19i3.3607.

UNITED STATES ENVIRONMENTAL PROTECTION AGENCY. Construction and demolition debris generation in the united states. 2016. Disponível em:

https://www.epa.gov/sites/production/files/2016-

12/documents/construction and demolition debris generation 201411302016 508.pdf. Acesso em: 11 mar. 2018.

VELTE, C. J.; STEINHILPER, R. Complexity in a Circular Economy: a need for rethinking complexity management strategies. In: WORD CONGRESS ON ENGINEERING.

[Proceedings...], 2016.

WORLD ECONOMIC FORUM. Towards the circular economy: accelerating the scale-up across global supply chains, 2014. Disponível em:

http://www3.weforum.org/docs/WEF ENV TowardsCircularEconomy Report 2014.pdf.

Acesso em: 10 mar. 2018.

WORLD ECONOMIC FORUM. Shaping the future of construction a breakthrough in mindset and technology. 2016. Disponível em:

http://www3.weforum.org/docs/WEF Shaping the Future of Construction full report .pdf. Acesso em: 10 mar. 2018.

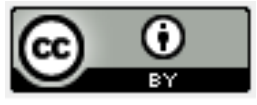

Artigo recebido em: 23/10/2019 e aceito para publicação em: 02/05/2020 DOI: http://dx.doi.org/10.14488/1676-1901.v20i2.3825 